\title{
Impaired VLDL assembly: a novel mechanism contributing to hepatic lipid accumulation following ovariectomy and high-fat/high-cholesterol diets?
}

\author{
Isabelle Côté ${ }^{1}$, Natalie A. Chapados ${ }^{2}$ and Jean-Marc Lavoie ${ }^{1 *}$ \\ ${ }^{1}$ Department of Kinesiology, Université de Montréal, 2100, Boulevard Édouard-Montpetit, Montréal, QC, Canada H3C 3J7 \\ ${ }^{2}$ Montfort Hospital Research Institute, Ottawa, ON, Canada
}

(Submitted 27 January 2014 - Final revision received 14 July 2014 - Accepted 22 July 2014 - First published online 29 September 2014)

\begin{abstract}
The aim of the present study was to identify molecular mechanisms involved in liver fat and cholesterol accumulation in ovariectomised (Ovx) rats fed with high-cholesterol diets. VLDL assembly and bile acid metabolism were specifically targeted. After being either Ovx or sham-operated, the rats were fed a standard diet or a high-fat diet containing $0,0.25$ or $0.5 \%$ cholesterol for 6 weeks. Although Ovx rats exposed to dietary cholesterol intake accumulated the greatest amount of hepatic fat and cholesterol, plasma cholesterol levels were lower $(P<0.05)$ in these animals than in the corresponding control rats. Accompanying this observation, ovariectomy and dietary cholesterol intake resulted in a down-regulation $(P<0.05)$ of the expression of genes associated with VLDL assembly, including microsomal TAG transfer protein, diacylglycerol acyltransferase 2, acyl-CoA:cholesterol acyltransferase 2 and apoB-100 as well as genes associated with bile acid metabolism including farnesoid $\mathrm{X}$ receptor and bile salt export pump $(P<0 \cdot 01)$. These results indicate that high-fat/high-cholesterol diets and ovariectomy concomitantly disrupt hepatic lipid output through defects in VLDL assembly and, most probably, secretion. The results also point to a defect in hepatic bile acid secretion. The present study offers novel insights into intrahepatic lipid metabolism, which may be relevant to metabolic complications found in postmenopausal women.
\end{abstract}

Key words: Dietary cholesterol: VLDL: Microsomal TAG transfer protein: Hepatic steatosis: Hepatic cholesterol accumulation

Excess dietary cholesterol consumption and oestrogen deficiency are two well-recognised independent factors inducing hepatic lipid accumulation ${ }^{(1-3)}$. There is also recent evidence that oestrogen deficiency and dietary cholesterol are two independent experimental variables that together potentiate hepatic lipid accumulation more than each one alone $^{(4)}$. Several pathways have been identified as deregulated by either dietary cholesterol or female hormone deficiency including hepatic inflammation ${ }^{(1,5)}$, decreased biliary cholesterol secretion ${ }^{(6)}$, stimulated de novo fatty acid synthesis ${ }^{(7)}$ and impaired VLDL production ${ }^{(8,9)}$, all contributing to the development of hepatic steatosis. Among them, VLDL secretion is crucial in preventing lipid accretion because the liver constantly takes up circulating lipids from both endogenous and exogenous sources ${ }^{(10,11)}$. However, molecular data supporting the defects in VLDL metabolism under the conditions of oestrogen deficiency and a hypercholesterolaemic diet are limited.

Although there are some physiological data supporting the concept that overconsumption of cholesterol induces the overproduction of hepatic lipoproteins ${ }^{(12,13)}$, other evidence points towards a reduction in VLDL assembly. For instance, we $^{(14)}$ and others ${ }^{(15)}$ found that a high-fat/high-cholesterol diet in rats suppressed the gene expression of microsomal TAG transfer protein (Mttp), a rate-limiting molecule for VLDL assembly and secretion. Dietary cholesterol has also been shown to increase cholesterol ester storage in hepatocytes and to reduce hepatic VLDL-TAG secretion, resulting in neutral lipid retention within the liver ${ }^{(11)}$. Similar to high cholesterol intake, oestrogen deficiency in ovariectomised (Ovx) animals or the blockage of oestrogen receptors results in a decrease of Mttp gene expression and impaired VLDLTAG secretion ${ }^{(8,9)}$.

Taking together, these findings suggest that the combination of ovariectomy and cholesterol consumption would disrupt VLDL assembly at the molecular level. To test this hypothesis, we measured the gene expression of several key molecular markers involved in the different steps of VLDL synthesis in Ovx rats fed with three diets rich in cholesterol. Besides Mttp, molecular markers of VLDL assembly included

Abbreviations: $C_{\mathrm{T}}$, threshold cycle; Cyp8b1, sterol $12 \alpha$-hydroxylase; Fxr, farnesoid X receptor; HF, high-fat diet; HFHC, high fat/high cholesterol; Ldlr, LDL receptor; Mttp, microsomal TAG transfer protein; Ovx, ovariectomised; SD, standard diet; SREBP-2, sterol regulatory element-binding protein 2; TC, total cholesterol.

* Corresponding author: J.-M. Lavoie, fax +1 514343 2181, email jean-marc.lavoie@umontreal.ca 
apoB-100, an essential structural protein that translocates into the luminal side of the endoplasmic reticulum ${ }^{(16,17)}$, diacylglycerol acyltransferase 2 (Dgat2), involved in converting fatty acids into TAG, and acyl-CoA:cholesterol acyltransferase 2 (Acat2) that converts free cholesterol into cholesterol esters $^{(18)}$. We also targeted cell death-inducing DNA fragmentation factor alpha (DFFA)-like effector B (Cideb), a lipid droplet-associated protein contributing to further lipidation of lipoprotein particles after they exit the endoplasmic reticulum compartment ${ }^{(19,20)}$, and small GTPase a (Sar1a), an intracellular vesicular trafficking protein that facilitates the movements of VLDL particles between the endoplasmic reticulum and the Golgi apparatus where they are secreted in the plasma.

Furthermore, we investigated the gene expression of the molecular markers for bile acid metabolism, a pathway that might also be involved in liver fat accumulation in Ovx rats under high dietary cholesterol feeding. These included farnesoid $\mathrm{X}$ receptor $(F x r)$, a nuclear receptor involved in hepatic bile acid metabolism ${ }^{(14)}$, and its target genes sterol $12 \alpha$-hydroxylase (Cyp8b1), which converts cholesterol into bile acids ${ }^{(21)}$, and bile salt export pump (Bsep), which stimulates bile acid excretion from the liver ${ }^{(22)}$

The aim of the present study was to identify novel mechanisms involved in hepatic steatosis induced by high dietary cholesterol consumption in Ovx rats. We found that in almost all the measured genes involved in VLDL assembly and bile acid metabolism, their transcripts were reduced by the combination of dietary cholesterol and oestrogen deficiency.

\section{Experimental methods}

\section{Animal care}

Female Sprague-Dawley rats ( $n$ 64) weighing 190-210 g were obtained from Charles River (St-Constant, PQ, Canada) and housed individually to monitor food intake in each animal. The $12 \mathrm{~h}$ light $-12 \mathrm{~h}$ dark cycle started at 06.00 hours, and room temperature was maintained at $20-23^{\circ} \mathrm{C}$. The animals had free access to food and water. Body weight and food intake were monitored twice per week. All the experiments in the present study were conducted according to the ARRIVE guidelines (BJN website; http://journals.cambridge. org/BJN) for animal research and the directives of the Canadian Council on Animal Care after institutional approval (CDEA: 12-108). The rat model and the number of rats ( $n 8$ per group) used in the present experiment have been repeatedly shown to be appropriate.

\section{Diets and surgery}

At 1 week after their arrival, the rats were either shamoperated ( $n$ 32) or Ovx ( $n$ 32) according to the technique described by Robertson et al. ${ }^{(23)}$. After surgery, the animals were injected with antibiotics (Tribrissen $24 \% ; 0 \cdot 125 \mathrm{~cm}^{3} / \mathrm{kg}$, subcutaneously) and analgesic (Carprofen; $4.4 \mathrm{mg} / \mathrm{kg}$, subcutaneously) for $3 \mathrm{~d}$. Thereafter, the Ovx and sham-operated rats were assigned one of the following four diets described in online supplementary Table S1.

\section{Blood and tissue sampling}

At 6 weeks after surgery, the rats were killed between 09.00 and 12.00 hours. Any remaining food was removed from the animal's cage at least $12 \mathrm{~h}$ before killing. Immediately after complete anaesthesia with isoflurane, the abdominal cavity was opened following the median line of the abdomen. Blood was collected into syringes treated with EDTA (15\%) and centrifuged $\left(3000 \mathrm{rpm} ; 4^{\circ} \mathrm{C} ; 10 \mathrm{~min}\right.$; Beckman GPR Centrifuge; Beckman Coulter). After blood collection, the liver median lobe was removed and freeze-clamped. The uterus, mesenteric, urogenital, retroperitoneal and subcutaneous fat deposits were removed and weighed (Mettler AE-100; Mettler Toledo). All tissues and plasma samples were stored at $-80^{\circ} \mathrm{C}$ until analyses.

\section{Molecular analyses}

Total RNA was extracted from the liver using RNA extraction Mini Kits (Invitrogen), according to the manufacturer's protocol. Thereafter, the RNA was treated with DNase (Invitrogen) to avoid genomic contamination. Total RNA $(2 \mu \mathrm{g})$ was reverse-transcribed into complementary DNA using highcapacity complementary DNA reverse transcription kits (Applied Biosystems). RT samples were stored at $-20^{\circ} \mathrm{C}$. The gene expression of the target genes was determined using assays designed with the Universal Probe Library. The primer sets and UPL probe numbers are presented in online supplementary Table S2. The ABI PRISM ${ }^{\circledR} 7900$ HT (Applied Biosystems) was used to detect the amplification level and programmed with an initial step of $3 \mathrm{~min}$ at $95^{\circ} \mathrm{C}$, followed by forty cycles for $5 \mathrm{~s}$ at $95^{\circ} \mathrm{C}$ and $30 \mathrm{~s}$ at $60^{\circ} \mathrm{C}$. All reactions were run in triplicate, and the average of threshold cycle $\left(C_{\mathrm{T}}\right)$ was used for quantification. The relative quantification of the target genes was determined using the $\Delta \Delta C_{\mathrm{T}}$ method. Briefly, the $C_{\mathrm{T}}$ values of the target genes were normalised to that of an endogenous control gene ( $\beta$-actin) $\left(\Delta C_{\mathrm{T}}=C_{\mathrm{T} \text { target }}-C_{\mathrm{T} \beta \text {-actin }}\right)$ and compared with a calibrator $\left(\Delta \Delta C_{\mathrm{T}}=\Delta C_{\mathrm{T} \text { Sample }}-\Delta C_{\mathrm{T} \text { Calibrator }}\right.$ ). Relative expression (RQ) was calculated using the Sequence Detection System 2.2.2 software (Applied Biosystems), and the formula is as follows: $\mathrm{RQ}=2^{-\Delta \Delta C_{\mathrm{T}}}$.

\section{Liver and plasma lipid measurements}

Liver TAG concentration was estimated from glycerol released after ethanolic $\mathrm{KOH}$ hydrolysis by a colorimetric method using commercial kits from Sigma. To measure liver cholesterol concentration, liver lipids were extracted using an adapted procedure developed by Folch et $a l .{ }^{(24)}$. Liver homogenate in a chloroform-methanol solution (2:1) was filtered and rinsed with chloroform. Methanol and water (20\% of the filtrate volume each) were added to the filtered solution. After vortexing, the solution with water and methanol was centrifuged for $20 \mathrm{~min}$ at $2400 \mathrm{rpm}$. The lower phase was transferred to clean tubes and evaporated overnight at $30^{\circ} \mathrm{C}$. The dried lipid residues were dissolved in $0.2 \mathrm{ml}$ of Triton X100isopropanol solution (10\%). Total cholesterol (TC) was 
measured with enzymatic kits (Wako). Plasma TC and TAG concentrations were measured using the COBAS INTEGRA 400 analyser (Roche Diagnostics).

\section{Statistical analysis}

Results are presented as means with their standard errors. Differences between means were tested for statistical significance $(P<0.05)$ using a two-way ANOVA for non-repeated measures with ovariectomy and diets as main factors. Fisher's least significant difference post hoc test was used in the event of a significant interaction effect $(P<0 \cdot 05)$. For a significant diet effect without interaction, Fisher's least significant difference from a one-way ANOVA was used.

\section{Results}

\section{Biometric parameters}

In all the dietary interventions, Ovx rats had higher food intake $(P<0.001)$ and changes in body composition including higher final body weight, intra-abdominal fat pads $(P<0.001)$ and subcutaneous adiposity $(P<0.05)$ compared with shamoperated animals (Table 1). A lower uterus weight in Ovx rats confirmed total ovariectomy compared with shamoperated rats (Table 1). The high-fat (HF) and highfat $+0.25 \%$ cholesterol (HFHC (0.25\%) diets did not affect intra-abdominal and subcutaneous fat deposits, food intake, and final body weight in both Ovx and sham-operated rats. However, sham-operated and Ovx rats fed the highfat $+0.5 \%$ cholesterol (HFHC (0.5\%)) diet had a higher food intake and final body weight $(P<0 \cdot 01$; Table 1$)$.

\section{Plasma lipids}

Plasma TAG concentrations were higher $(P<0.05)$ upon ovariectomy in all the dietary interventions (Fig. 1(a)). Surprisingly, the HFHC diet-fed rats exhibited lower plasma TAG concentrations $(P<0.001)$ than their corresponding standard diet (SD)-fed rats (Fig. 1(a)). In contrast, the effect of ovariectomy on plasma TC concentrations was largely influenced by the dietary interventions. After feeding the SD, plasma TC concentrations were higher by $64 \%(P<0.01)$ in Ovx rats than in sham-operated rats, but the differences between the Ovx and sham-operated rats were attenuated after feeding the HF and HFHC (0.25\%) diets (Fig. 1(b)). Surprisingly, increasing the dietary cholesterol content to $0.5 \%$ (HFHC) reversed the effect of ovariectomy on plasma TC concentrations reported in SD-fed animals. Indeed, in response to the HFHC (0.5\%) diet, plasma TC concentration in Ovx rats was $28 \%$ lower than that in the corresponding sham-operated rats $(2.72 v .3 .74 \mathrm{mmol} / 1 ; P<0.05)$. By comparison, plasma TC concentrations in response to the two HFHC diets increased more linearly in sham-operated rats than in Ovx rats, with increases reaching up to $56 \%(0.25 \%$ cholesterol $)$ and $113 \%$ ( $0.5 \%$ cholesterol) compared with SD-fed animals $(P<0 \cdot 01$; Fig. 1(b)). 
(a)

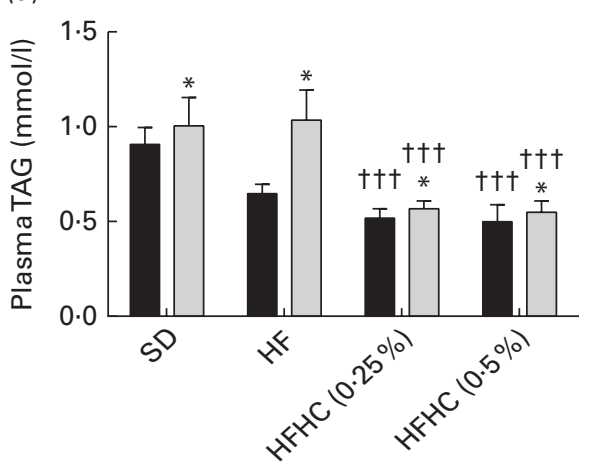

(c)

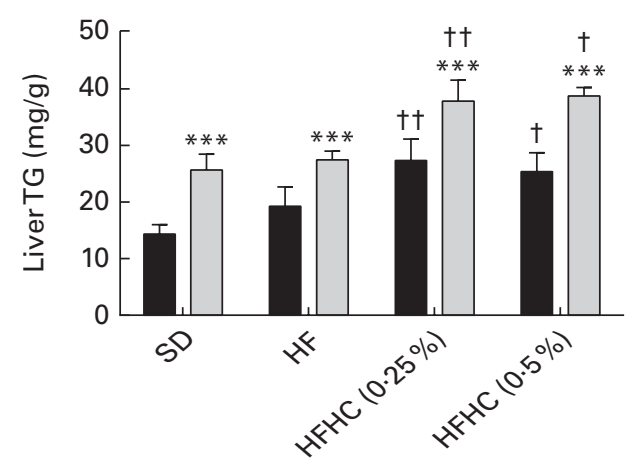

(b)

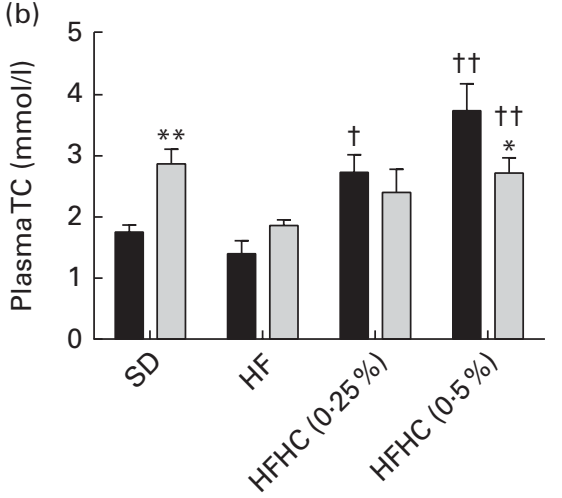

(d)

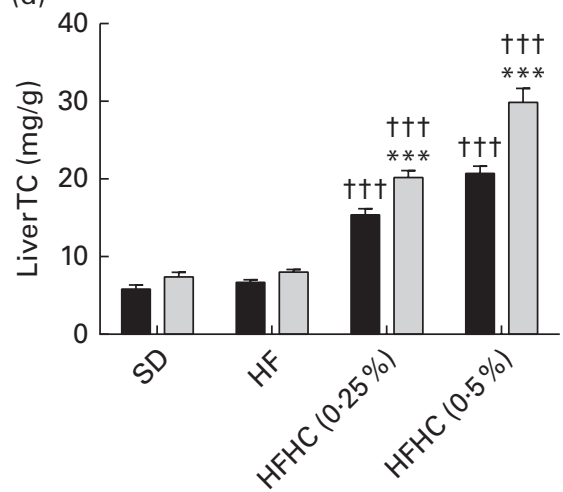

Fig. 1. Plasma and hepatic TAG and total cholesterol (TC) in sham-operated ( $\square$ ) and ovariectomised (Ovx, $\square$ ) rats fed either a standard diet (SD), a high-fat (HF) diet, a HF $+0.25 \%$ cholesterol (HFHC $(0.25 \%)$ ) diet or a HF $+0.5 \%$ cholesterol (HFHC $(0.5 \%)$ ) diet. Values are means, with their standard errors represented by vertical bars. Mean value was significantly different from that of the sham-operated rats: ${ }^{*} P<0.05,{ }^{* \star} P<0.01,{ }^{* \star *} P<0.001$ (ovariectomy as the main effect). Mean value was significantly different from that of the SD: $\dagger P<0.05$, $\uparrow \dagger P<0.01, \dagger_{\dagger}+P<0.001$ (diet as the main effect).

\section{Liver lipids}

As expected, Ovx rats had higher liver TAG concentrations (37-78\%; $P<0.001)$ in all the dietary interventions than the corresponding sham-operated rats (Fig. 1(c)). High cholesterol consumption in sham-operated rats was associated with $90 \%$ $(P<0.01 ; 0.25 \%)$ and $76 \%(P<0.05 ; 0.5 \%)$ higher liver TAG content than that in SD-fed rats. Interestingly, ovariectomy and HFHC diets concomitantly induced a higher level of hepatic steatosis. In fact, liver TAG levels in HFHC diet-fed Ovx animals reached up to $151 \%$ of the levels found in SD-fed Ovx rats and up to $270 \%$ of the levels measured in SD-fed sham-operated rats (Fig. 1(c)). Liver TC content was not increased in Ovx rats compared with sham-operated rats when fed the SD and the HF diet (Fig. 1(d)). However, after feeding both HFHC diets, liver TC content in Ovx rats was $131 \%(P<0.001 ; 0.25 \%)$ and $144 \%(P<0.001 ; 0.5 \%)$ higher than the values measured in sham-operated rats (Fig. 1(d)). In response to the HFHC diets, hepatic TC levels in shamoperated rats were $170 \%(P<0.001 ; 0.25 \%)$ and $280 \%$ $(P<0.001 ; 0.5 \%)$ of the levels reported in SD-fed shamoperated animals. In Ovx rats fed the two HFHC diets, liver TC content values reached up to $270-400 \%(P<0 \cdot 01)$ of the values measured in SD-fed Ovx rats and 350-520\% $(P<0 \cdot 001)$ of the values measured in SD-fed sham-operated rats, indicating a concomitant increase (Fig. 1(d)).

\section{Molecular markers of VLDL assembly and secretion}

The gene expression levels of Mttp and Dgat2, two key molecules in VLDL assembly, were lower $(P<0.001)$ in Ovx animals, regardless of the dietary interventions (Fig. 2(a) and (b)). In addition, the gene expression levels of Apob-100, Acat2, Sar1a and Cideb, also involved in VLDL synthesis and secretion, were lower $(P<0.001)$ in Ovx rats than in sham-operated rats in all the dietary interventions (Fig. 2(c)-(f)). In sham-operated and Ovx rats, both HFHC diets were associated with a lower gene expression level $(P<0 \cdot 001)$ of Mttp (Fig. 2(a)). Considering the cumulative effects, the HFHC-fed Ovx rats showed a large decrease in the gene expression of $M t t p$ such that the levels dropped as low as $31 \%$ of those reported in SD-fed sham-operated rats.

\section{Molecular markers of hepatobiliary cholesterol and bile acid excretion}

The gene expression of nuclear receptors Fxr and liver $\mathrm{X}$ receptor $($ Lxr $)$ was lower $(P<0.05$ and $<0.001)$ in Ovx rats than in sham-operated animals, in all the dietary interventions (Fig. 3(a) and (b)). In Ovx and sham-operated rats, both HFHC diets decreased the gene expression level of Fxr $(P<0.01)$, but the transcript level of $L x r$ was decreased only in HFHC $(0.5 \%)$-fed rats $(P<0.01)$. Regardless of the dietary 

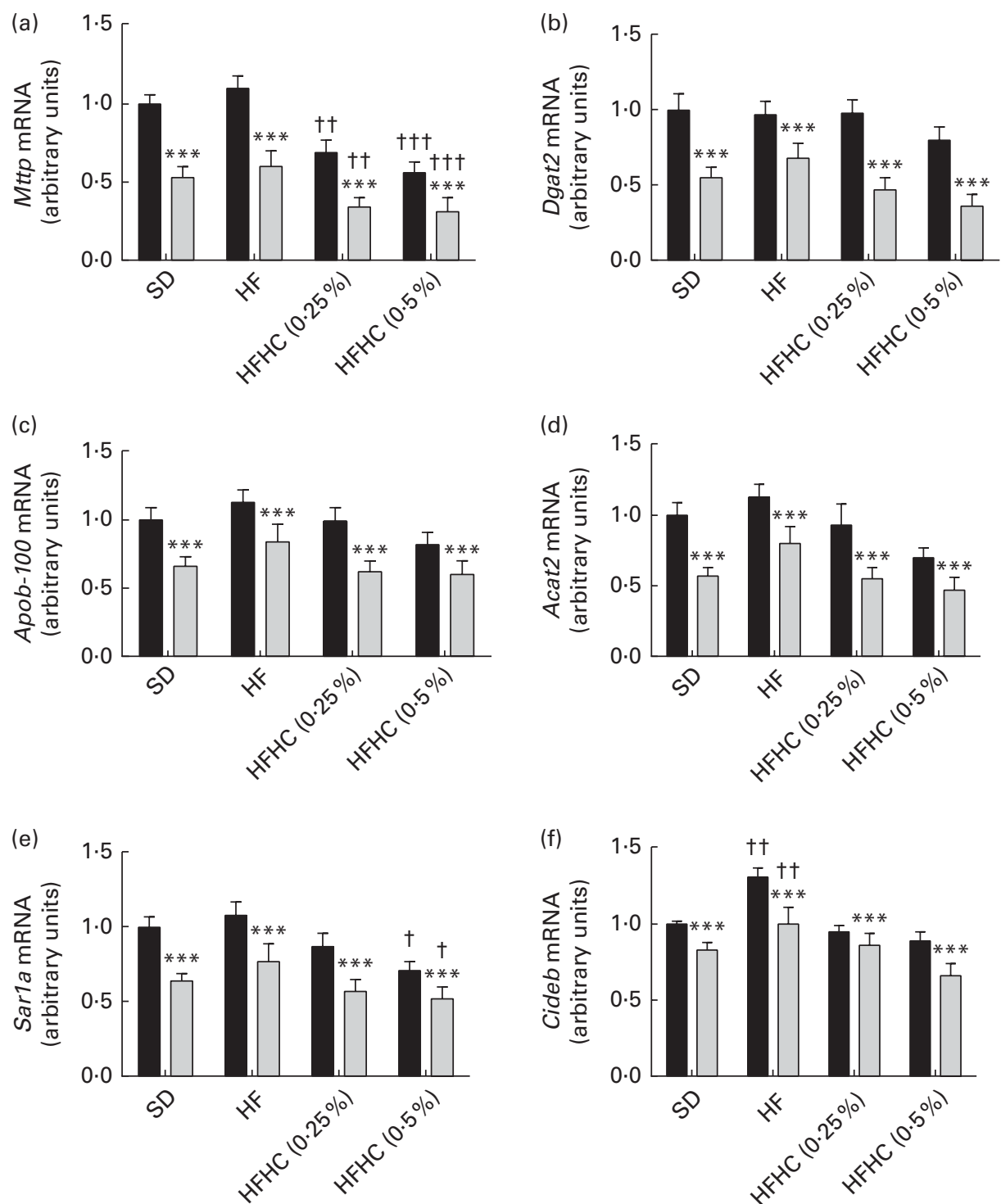

Fig. 2. Hepatic gene expression of microsomal TAG transfer protein (Mttp), diacylglycerol acyl transferase 2 (Dgat2), Apob-100, acyl-CoA cholesterol acyl transferase 2 (Acat2), small GTP-binding protein a (Sar1a) and cell death-inducing DNA fragmentation factor alpha (DFFA)-like effector type B (Cideb) in sham-operated $(\square)$ or ovariectomised (Ovx, $\square$ ) rats fed either a standard diet (SD), a high-fat (HF) diet, a HF $+0.25 \%$ cholesterol (HFHC (0.25\%)) diet or $\mathrm{HF}+0.5 \%$ cholesterol (HFHC $(0.5 \%)$ ) diet. Values are means, with their standard errors represented by vertical bars. ${ }^{* * *}$ Mean value was significantly different from that of the sham-operated rats $(P<0.001$; ovariectomy as the main effect). Mean value was significantly different from that of the SD: $† P<0.05$, †† $P<0.01$, †† $P<0.001$ (diet as the main effect).

interventions, oestrogen deficiency was associated with lower transcript levels of hepatic Cyp8b1 and Bsep, suggesting that the synthesis and excretion of bile acids were decreased in Ovx rats (Fig. 3(c) and (d)). In addition, the two HFHC diets further decreased the gene expression level of Cyp $8 b 1$ $(P<0.001)$ in sham-operated rats as well as in Ovx rats, but the expression level of Bsep was not affected by these dietary interventions (Fig. 3(c)). Finally, the transcript levels of the canalicular cholesterol excretion transporters ATP-cassette binding protein G5/G8 (ABCG5/ABCG8) were not affected by ovariectomy. However, the gene expression of Abcg5/ Abcg8 was 2- to 3-fold higher $(P<0 \cdot 01)$ in both Ovx and sham-operated rats fed the HF diet than in those fed the SD.

\section{Molecular markers of hepatic cholesterol metabolism}

The gene expression levels of sterol regulatory elementbinding protein 2 (Srebp2), a key regulator of hepatic cholesterol content, and its target genes LDL receptor $(L d l r)$ and 3-hydroxy-3-methyl-glutaryl-CoA reductase (Hmgcr) were down-regulated $(P<0.001)$ in Ovx rats compared with shamoperated rats after the SD and HF dietary interventions (Fig. 4(a) and (c)). In sham-operated rats, the HF diet did not affect the gene expression levels of Srebp2 and $L d l r$, but stimulated $(P<0.05)$ the expression level of $\mathrm{Hmgcr}$ (Fig. 4(a) and (c)). However, the addition of cholesterol to the HF diet highly suppressed the gene expression levels of Srebp2 $(P<0.01), L d l r(P<0.001)$ and Hmgcr $(P<0.05)$ in 

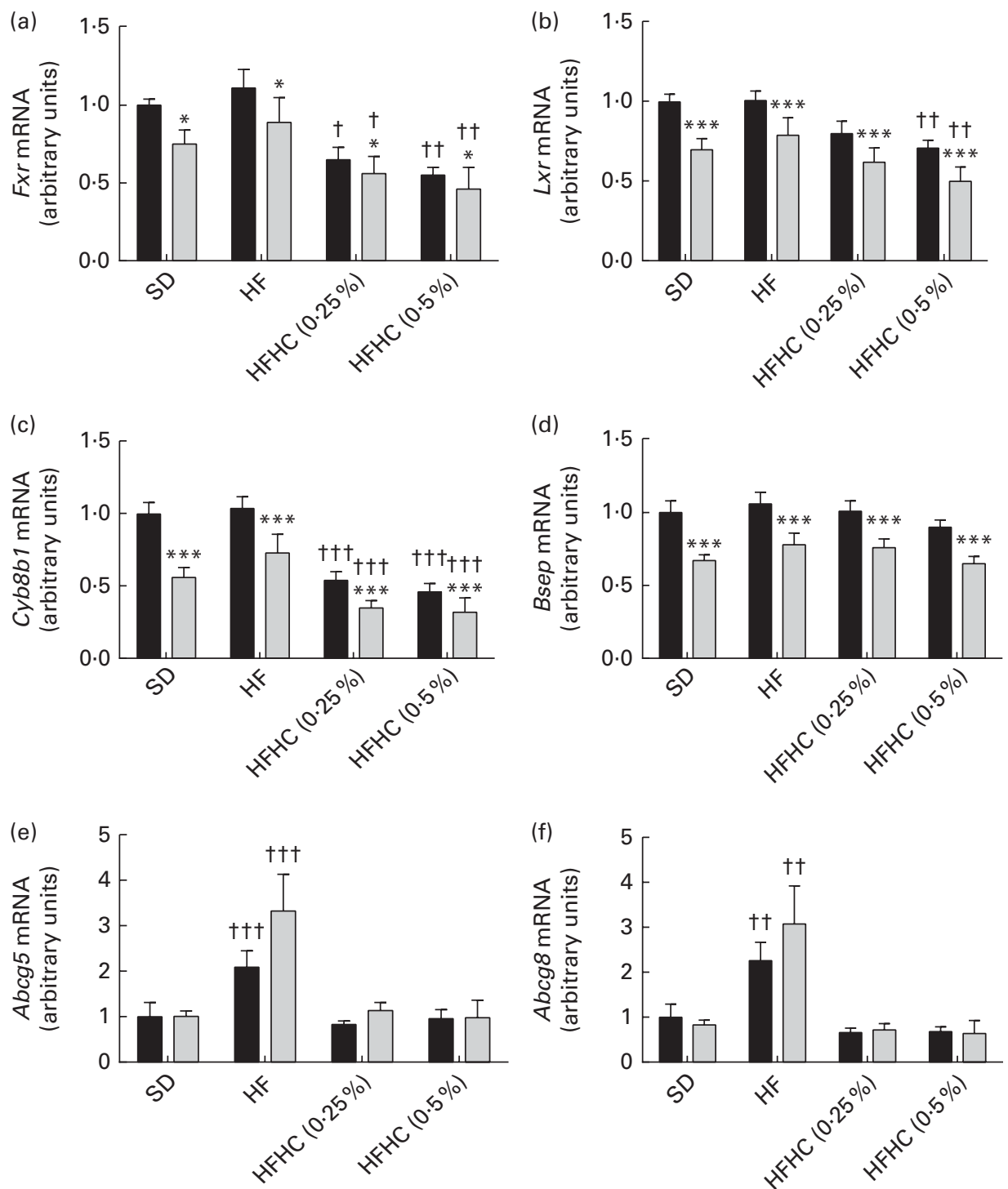

Fig. 3. Hepatic gene expression of farnesoid $X$ receptor (Fxr), liver $X$ receptor (Lxr), sterol 12 $\alpha$-hydroxylase (Cyp8b1), bile salt export pump (Bsep), ATPcassette binding protein G5 and G8 (Abcg5/Abcg8) in sham-operated ( $\square$ ) or ovariectomised (Ovx, $\square$ ) rats fed either a standard diet (SD), a high-fat (HF) diet, a $\mathrm{HF}+0.25 \%$ cholesterol (HFHC $(0.25 \%)$ ) diet or a HF $+0.5 \%$ cholesterol (HFHC $(0.5 \%)$ ) diet. Values are means, with their standard errors represented by vertical bars. Mean value was significantly different from that of the sham-operated rats: ${ }^{*} P<0.05,{ }^{* * *} P<0.001$ (ovariectomy as the main effect). Mean value was significantly different from that of the SD: $\uparrow P<0.05, \dagger^{\dagger} P<0.01, \uparrow_{\dagger} P<0.001$ (diet as the main effect).

sham-operated rats. However, the ingestion of the HFHC diets did not further decrease the gene expression levels of Srebp2, Ldlr and Hmgcr in Ovx rats (Fig. 4(c) and (d)).

\section{Discussion}

The aim of the present study was to identify novel mechanisms involved in hepatic steatosis induced by a HFHC diet and oestrogen deficiency in rats. Although the relevance of the present data to postmenopausal women needs to be established, to our knowledge, the present study is the first to provide hepatic and plasma lipid profiles accompanied by molecular changes that together indicate a defect in VLDL assembly upon ovariectomy and/or HFHC diet consumption. Additionally, our molecular analyses revealed a second potential novel mechanism through which ovariectomy and HFHC diets may collectively enhance hepatic steatosis, namely defective hepatic bile acid secretion.

\section{Ovariectomy and high-fat/high-cholesterol diets concomitantly repressed VLDL assembly-related enzymes}

The present study corroborated the synergistic effect of high cholesterol consumption and the oestrogen deficiency state by ovariectomy on the development of hepatic steatosis. We found that liver TAG concentration was higher by 37 to $78 \%$ in all Ovx rats compared with sham-operated rats fed the corresponding diets. More importantly, the highest liver TAG levels were observed in Ovx rats fed the HFHC diets, reaching up to $270 \%$ of the level measured in SD-fed sham-operated 

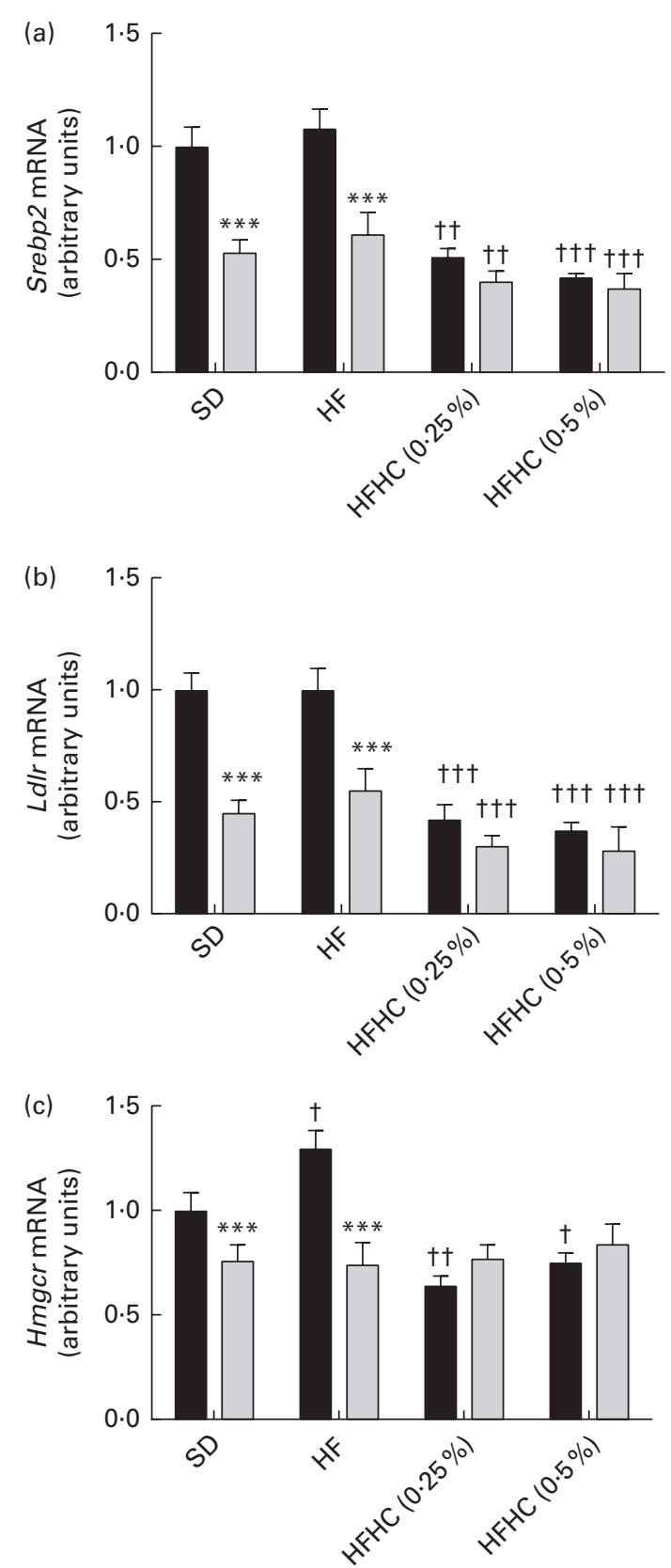

Fig. 4. Hepatic gene expression of sterol regulatory element-binding protein-2 (Srebp2), LDL receptor (Ldlr) and hydroxy-3-methyl-glutaryl-CoA reductase $(\mathrm{Hmgcr})$ in sham-operated $(\square)$ or ovariectomised $(\mathrm{Ovx}, \square)$ rats fed either a standard diet (SD), a high-fat (HF) diet, a $\mathrm{HF}+0.25 \%$ cholesterol (HFHC $(0.25 \%)$ ) diet or a $\mathrm{HF}+0.5 \%$ cholesterol (HFHC $(0.5 \%)$ ) diet. Values are means, with their standard errors represented by vertical bars. ${ }^{* * *}$ Mean value was significantly different from that of the sham-operated rats $(P<0.001$; ovariectomy as the main effect). Mean values were significantly different from that of the SD: $\dagger P<0.05$, $\dagger \dagger P<0.01$, $\dagger+\dagger P<0.001$ (diet as the main effect).

rats. We observed that Ovx rats compared with sham-operated rats presented substantially lower gene expression levels of Acat2 and Dgat2, enzymes involved in the synthesis of cholesterol esters and TAG, respectively, which are the two components of the VLDL lipid core ${ }^{(25,26)}$. In addition, the gene expression of $M t t p$, a determinant enzyme that interacts with the N-terminus of apoB allowing initial lipid transfer into nascent VLDL particles, was lower in Ovx rats than in sham-operated rats ${ }^{(16)}$. Finally, Cideb, a molecule recently identified as critical in VLDL lipidation, and Sar1 GTPase, involved in VLDL transfer from the endoplasmic reticulum to the Golgi apparatus, were also lower in Ovx rats than in sham-operated rats ${ }^{(19)}$. Collectively, these results are consistent with the interpretation that VLDL assembly is disrupted upon ovariectomy.

Besides the effects of ovariectomy, it appears that HFHC feeding also disrupted VLDL assembly and secretion. The gene expression of Mttp measured in all HFHC-fed rats was decreased to the levels as low as $31 \%$ of those reported in SD-fed sham-operated rats (Fig. 2(a)). Similar decreases in the gene expression of Mttp following the HFHC diets have been previously reported by us ${ }^{(14)}$ and others ${ }^{(15)}$. In addition, we found that plasma TAG levels were largely reduced in sham-operated and Ovx rats fed the HFHC diets. Since the liver provides the major source of plasma TAG under the fasted state, it has been reported that fasted plasma TAG level can be considered as an indirect marker for hepatic VLDL production ${ }^{(27)}$. Therefore, lower levels of fasted plasma TAG found in sham-operated and HFHC-fed Ovx animals further support the possibility that HFHC feeding impairs liver VLDL secretion. Taken together, the present results suggest that impaired VLDL production contributes to hepatic lipid accumulation in Ovx rats as well as in all rats fed the HFHC diets. Moreover, disruption of VLDL production is a mechanism that may explain the high accumulation of TAG found in the liver of Ovx rats fed the HFHC diets compared with those fed the other diets.

\section{Ovariectomy and high-fat/high-cholesterol diets down-regulated the gene expression of bile acid excretion}

An additional plausible explanation for hepatic cholesterol accumulation in Ovx rats fed the HFHC diets is the decrease in the synthesis and excretion of biliary acids. This view is supported by the observation that animals fed the HFHC diets had a lower gene expression level of Fxr, the regulator of hepatic bile acid metabolism, and its target gene Cyp $8 b 1$. The specific role of hepatic Fxr is to initiate the expression of a complete gene network involved in the synthesis and excretion of bile acids in order to prevent bile acid hepatotoxicity. Since bile acids are synthesised from cholesterol in hepatocytes, bile acid synthesis is also an important pathway to remove cholesterol from the liver $^{(28)}$. Furthermore, bile acid secretion exerts the driving force for biliary cholesterol excretion, another pathway for liver cholesterol output ${ }^{(29,30)}$. The role of bile acids on liver lipids and cholesterol metabolism has been enlightened by the generation of Fxr-null mice, showing massive accumulation of TAG and cholesterol in hepatocytes ${ }^{(31)}$. Dietary interventions, such as HFHC diets, have also been reported to repress the gene expression of hepatic $\operatorname{Fxr}^{(14,15)}$. The present decrease in the gene expression levels of Fxr and Cyp8b1 in Ovx rats fed with the HFHC diets may thus be taken as an indication that bile acid metabolism may be reduced and, in turn, favours cholesterol accumulation in the liver. 
Disruption in bile acid metabolism might also be involved in higher TC accumulation found in the liver of Ovx rats compared with sham-operated rats. Ovariectomy suppressed the transcription factors Fxr and $L x r$ as well as Cyp8b1 and Bsep, enzymes involved in bile acid synthesis and excretion, respectively. In line with these results, Czerny et al. ${ }^{(32-34)}$ found a significant decrease in total bile production in Ovx rats, thus supporting the hypothesis that biliary metabolic pathways are disrupted in Ovx animals. Since disrupted biliary metabolic pathways restrain hepatic cholesterol output, the repression of key enzymes involved in bile acid excretion by ovariectomy as well as by HFHC diet consumption is consistent with the massive accumulation of liver TC in Ovx rats fed the HFHC diets.

\section{Ovariectomy and high-fat/high-cholesterol diets repressed hepatic cholesterol regulatory molecules}

Liver TC content was not changed in Ovx rats compared with sham-operated rats after the SD and HF diet interventions, corroborating previous reports ${ }^{(4,35)}$. However, liver TC levels accumulated in both Ovx and sham-operated rats following the HFHC diets and to a larger extent in Ovx rats than in sham-operated rats, indicating a synergistic action of these two interventions (Fig. 1). Liver TC levels accumulated in these rats despite the fact that cholesterol synthesis, as supported by the transcript levels of Hmgcr, was reduced in Ovx rats and by the HFHC diets in sham-operated animals. An increase in TC content in the liver of Ovx rats has also been associated with a lower gene expression level of the transcription factor Srebp2 and its target gene $L d l r^{(36)}$. Accordingly, the mRNA levels of Srebp 2 and $L d l r$ were decreased following ovariectomy and HFHC diet consumption in the present study. The lower expression of $L d l r$ may explain the hypercholesterolaemia observed in Ovx rats following the SD and HF dietary interventions. In contrast, the higher liver cholesterol levels found in Ovx rats compared with sham-operated rats fed the HFHC diets might be associated with a reduction in VLDL synthesis and secretion, as mentioned previously.

\section{Ovariectomy and high-fat/high-cholesterol diets differently affected plasma lipid profile}

As reported previously ${ }^{(37)}$, Ovx rats had significantly higher plasma TC concentrations than sham-operated rats following the SD. Unexpectedly, this difference between the Ovx and sham-operated animals was entirely abolished after consumption of the HF and HFHC diets. An intriguing outcome was that following the HFHC diets, the difference between the Ovx and sham-operated rats on plasma cholesterol concentration was even reversed, with higher levels being found in sham-operated rats than in Ovx rats. Given that these lower plasma cholesterol levels in Ovx rats following the HFHC diets are accompanied by higher liver TC levels, it may be deduced that ovariectomy led to the impaired mobilisation of cholesterol from the liver.
In summary, the results of the present study indicate that HFHC diets and ovariectomy concomitantly stimulated hepatic lipid and cholesterol accumulation. Molecular changes observed in VLDL assembly and bile acid regulation of key molecules suggest that HFHC diets and ovariectomy both affect hepatic lipid retention through a decrease in VLDL assembly and bile acid synthesis.

\section{Supplementary material}

To view supplementary material for this article, please visit http://dx.doi.org/10.1017/S0007114514002517

\section{Acknowledgements}

The present study was supported by grants from the Natural Sciences and Engineering Research Council of Canada (NSERC; 7594) and from the Canadian Institutes of Health Research (CIHR; T 0602 145.02).

The authors' contributions are as follows: I. C. was responsible for the conception, design and acquisition of the data; N. A. C. was involved in the acquisition of the data; J.-M. L. contributed to the conception and design of the study. All authors interpreted the data, drafted/revised the manuscript for important intellectual content, and approved the final version.

The authors declare that they have no conflicts of interest.

\section{References}

1. Subramanian S, Goodspeed L, Wang S, et al. (2011) Dietary cholesterol exacerbates hepatic steatosis and inflammation in obese LDL receptor-deficient mice. J Lipid Res 52, 1626-1635.

2. Comhair TM, Garcia Caraballo SC, Dejong CH, et al. (2011) Dietary cholesterol, female gender and $n-3$ fatty acid deficiency are more important factors in the development of non-alcoholic fatty liver disease than the saturation index of the fat. Nutr Metab (Lond) $\mathbf{8}, 4$.

3. Kainuma M, Fujimoto M, Sekiya N, et al. (2006) Cholesterolfed rabbit as a unique model of nonalcoholic, nonobese, non-insulin-resistant fatty liver disease with characteristic fibrosis. J Gastroenterol 41, 971-980.

4. Kamada Y, Kiso S, Yoshida Y, et al. (2011) Estrogen deficiency worsens steatohepatitis in mice fed high-fat and high-cholesterol diet. Am J Physiol Gastrointest Liver Physiol 301, G1031-G1043.

5. Dimitrova-Shumkovska J, Veenman L, Ristoski T, et al. (2010) Chronic high fat, high cholesterol supplementation decreases $18 \mathrm{kDa}$ Translocator Protein binding capacity in association with increased oxidative stress in rat liver and aorta. Food Chem Toxicol 48, 910-921.

6. Treguier M, Briand F, Boubacar A, et al. (2011) Diet-induced dyslipidemia impairs reverse cholesterol transport in hamsters. Eur J Clin Invest 41, 921-928.

7. Fungwe TV, Fox JE, Cagen LM, et al. (1994) Stimulation of fatty acid biosynthesis by dietary cholesterol and of cholesterol synthesis by dietary fatty acid. J Lipid Res 35, 311-318.

8. Cote I, Yasari S, Pighon A, et al. (2012) Liver fat accumulation may be dissociated from adiposity gain in ovariectomized rats. Climacteric 15, 594-601. 
9. Barsalani R, Chapados NA \& Lavoie JM (2010) Hepatic VLDL-TG production and MTP gene expression are decreased in ovariectomized rats: effects of exercise training. Horm Metab Res 42, 860-867.

10. Flamment M, Kammoun HL, Hainault I, et al. (2010) Endoplasmic reticulum stress: a new actor in the development of hepatic steatosis. Curr Opin Lipidol 21, 239-246.

11. Alger HM, Brown JM, Sawyer JK, et al. (2010) Inhibition of acyl-coenzyme A:cholesterol acyltransferase 2 (ACAT2) prevents dietary cholesterol-associated steatosis by enhancing hepatic triglyceride mobilization. J Biol Chem 285 , 14267-14274

12. Teramoto T, Kato H, Hashimoto $\mathrm{Y}$, et al. (1987) Effect of dietary cholesterol on production of lipoproteins and apolipoproteins by perfused livers from Japanese monkeys (Macaca fuscata). Eur J Clin Invest 17, 522-529.

13. Chen J, Song W \& Redinger RN (1996) Effects of dietary cholesterol on hepatic production of lipids and lipoproteins in isolated hamster liver. Hepatology 24, 424-434.

14. Cote I, Ngo Sock ET, Levy E, et al. (2013) An atherogenic diet decreases liver FXR gene expression and causes severe hepatic steatosis and hepatic cholesterol accumulation: effect of endurance training. Eur J Nutr 52, 1523-1532.

15. Savard C, Tartaglione EV, Kuver R, et al. (2013) Synergistic interaction of dietary cholesterol and dietary fat in inducing experimental steatohepatitis. Hepatology 57, 81-92.

16. Hussain MM, Bakillah A, Nayak N, et al. (1998) Amino acids 430-570 in apolipoprotein B are critical for its binding to microsomal triglyceride transfer protein. J Biol Chem 273, $25612-25615$.

17. Hussain MM, Shi J \& Dreizen P (2003) Microsomal triglyceride transfer protein and its role in apoB-lipoprotein assembly. J Lipid Res 44, 22-32.

18. Cianflone KM, Yasruel Z, Rodriguez MA, et al. (1990) Regulation of apoB secretion from HepG2 cells: evidence for a critical role for cholesteryl ester synthesis in the response to a fatty acid challenge. J Lipid Res 31, 2045-2055.

19. Tiwari S, Siddiqi S \& Siddiqi SA (2013) CideB protein is required for the biogenesis of very low density lipoprotein (VLDL) transport vesicle. J Biol Chem 288, 5157-5165.

20. Ye J, Li JZ, Liu Y, et al. (2009) Cideb, an ER- and lipid droplet-associated protein, mediates VLDL lipidation and maturation by interacting with apolipoprotein B. Cell Metab 9, 177-190.

21. Russell DW (2003) The enzymes, regulation, and genetics of bile acid synthesis. Annu Rev Biochem 72, 137-174.

22. Alrefai WA \& Gill RK (2007) Bile acid transporters: structure, function, regulation and pathophysiological implications. Pharm Res 24, 1803-1823.

23. Robertson MC, Owens RE, Klindt J, et al. (1984) Ovariectomy leads to a rapid increase in rat placental lactogen secretion. Endocrinology 114, 1805-1811.
24. Folch J, Lees M \& Sloane Stanley GH (1957) A simple method for the isolation and purification of total lipides from animal tissues. J Biol Chem 226, 497-509.

25. Willner EL, Tow B, Buhman KK, et al. (2003) Deficiency of acyl CoA:cholesterol acyltransferase 2 prevents atherosclerosis in apolipoprotein E-deficient mice. Proc Natl Acad Sci U S A 100, 1262-1267.

26. Liu Y, Millar JS, Cromley DA, et al. (2008) Knockdown of acyl-CoA:diacylglycerol acyltransferase 2 with antisense oligonucleotide reduces VLDL TG and ApoB secretion in mice. Biochim Biophys Acta 1781, 97-104.

27. Bjorkegren J, Karpe F, Milne RW, et al. (1998) Differences in apolipoprotein and lipid composition between human chylomicron remnants and very low density lipoproteins isolated from fasting and postprandial plasma. J Lipid Res 39, $1412-1420$

28. Vlahcevic ZR (1996) Regulation of cholesterol 7 alphahydroxylase by different effectors. Ital J Gastroenterol $\mathbf{2 8}$, 337-339.

29. Oude Elferink RP, Paulusma CC \& Groen AK (2006) Hepatocanalicular transport defects: pathophysiologic mechanisms of rare diseases. Gastroenterology 130, 908-925.

30. Oude Elferink RP \& Groen AK (2000) Mechanisms of biliary lipid secretion and their role in lipid homeostasis. Sem Liver Dis 20, 293-305.

31. Sinal CJ, Tohkin M, Miyata M, et al. (2000) Targeted disruption of the nuclear receptor FXR/BAR impairs bile acid and lipid homeostasis. Cell 102, 731-744.

32. Czerny B, Teister M, Juzyszyn Z, et al. (2011) The effect of retinoic acid receptor agonist acitretin on the production of bile and concentrations of some serum components in ovariectomized rats. Menopause 18, 213-218.

33. Czerny B, Teister M, Juzyszyn Z, et al. (2006) Effect of tamoxifen and raloxifene on the conjugation of bile acids with taurine and glycine in ovariectomized rats. Pharmacol Rep 58, 435-438.

34. Czerny B, Teister M, Juzyszyn Z, et al. (2005) Effect of tibolone on turnover of cholesterol to bile acids in ovariectomized rats. Menopause 12, 609-612.

35. Kato M, Ogawa H, Kishida T, et al. (2009) The mechanism of the cholesterol-lowering effect of water-insoluble fish protein in ovariectomised rats. Br J Nutr 102, 816-824.

36. Ngo Sock ET, Chapados NA \& Lavoie JM (2014) LDL receptor and Pcsk9 transcripts are decreased in liver of ovariectomized rats: effects of exercise training. Horm Metab Res 46, 550-555.

37. Lucas EA, Mahajan SS, Soung do Y, et al. (2011) Flaxseed but not flaxseed oil prevented the rise in serum cholesterol due to ovariectomy in the Golden Syrian hamsters. J Med Food 14, 261-267. 